\title{
The Expression of MAP2 in the Process of PC12 Cells Differentiated into Neurons
}

\author{
Lijuan Zhang ${ }^{1, a}$, Chunli Mei ${ }^{1, b^{*}}$ and Zhenwei Fan ${ }^{1, c}$ \\ Beihua University , Jilin, 132013, China \\ a514324085@qq.com, bmeixiaoqing2007@126.com, '22289671@qq.com \\ ${ }^{*}$ Chunli Mei is the Corresponding Author
}

\begin{abstract}
Keywords: MAP2 expression,PC12 cells, Time points
Abstract. MAP2 plays a vital role in PC12 cell differentiation, expecialy the length of the synapse and the formation of microtubules. Here, NGF (Nerve Growth Factor) and OGD (oxygen-glucose deprivation) was used to stimulate PC12 cells, and convert them into neurons to establish an ischemia in vitro model. In order to further find MAP2 expression in PC12 cells. Immunocytochemical analysis and western blot were used to determinate the protein expression of MAP2. MAP2 expression was determined by western blotting after different treatment time periods. The results showed three repeated experiments. The expression of MAP2 was different in different time. Consult the relevant literature, PC12 cells can be inducted into Nerve cells by NGF. MAP2 exists in PC12 cell differentiation time point. It is closely related with the length of the synapses and microtubules formation. MAP2 plays an important role in synaptic neuron growth.
\end{abstract}

\section{Introduction}

Ischemic stroke is a serious harm human health disease, its mortality and morbidity is high and explore its pathogenesis and effective prevention and treatment has been the hot topics in the study of medical workers. In ischemic cerebral apoplexy, in order to look for can be applied in clinical nerve protection material targets and key role in signal transduction pathways, molecular level research [1].

MAP2 exists in PC12 cell differentiation process, it has to do with the length of the synaptic and closely related to the formation of microtubules, MAP2 in neurons synaptic growth plays an important role in [2-3]. In the present study, we use the immune fluorescence to detect the expression of MAP2 and Western blot detection model NGF stimuli changes the expression of MAP2 in PC12 cells in different time points, the results showed that the expression of MAP2 with increasing trend with the extension of NGF plays the induction time. Description model NGF induced PC12 cells to differentiate into nerve cells, the protein expression of MAP2 in dynamic change. This provides the study of ischemic cerebral apoplexy cell basis.

\section{Materials and Methods}

Cell Culture and Oxygen-glucose Deprivation PC12 cells were purchased from the Cell Home of the Chinese Academy of Sciences. The cells were cultured in the DMEM supplemented with the 10\% (v/v) fetal bovine serum (FBS, GIBCO). Cells were treated with $100 \mathrm{ng} / \mathrm{mL}$ nerve growth factor (NGF 2.5S; Promega, Madison, WI) for 24 hours. After that, the cells were transfected with promoter constructs and cultured for additional 5 days in the medium containing NGF. PC12 cells were treated with NGF for 6 days. Then the cells were washed 3 times with DMEM, and cultured with DMEM in the presence of no sugar and $1 \mathrm{mmol} / \mathrm{L} \mathrm{Na} 2 \mathrm{~S} 2 \mathrm{O} 4$ in hypoxic conditions $\left(37^{\circ} \mathrm{C}, 5 \% \mathrm{CO} 2\right.$ and $\left.95 \% \mathrm{~N} 2\right)$ for $16 \mathrm{~h}[4]$.

Map2 Immunocytochemical Analysis The cells were fixed with 4\% paraformaldehyde/PBS and were permeabilized with $0.1 \%$ Triton X-100 in PBS for $10 \mathrm{~min}$. Then the cells were incubated in 5\% goat serum/PBS for $1 \mathrm{~h}$ at room temperature. Cells were then washed again, and incubated overnight at $4^{\circ} \mathrm{C}$ in the presence of anti-Map2 (1:1,000 dilution, Santa). After washing twice with PBS, the cells 
were incubated with fluorescently labeled secondary FITC-rabbit anti-goat (Santa) for $1 \mathrm{~h}$ at room temperature. The results were observed witha fluorescence microscope equipped with a photomicrographic system [5].

Western Blotting Analysis After treatment with NGF 6 days and OGD for 16 hours, the cells were

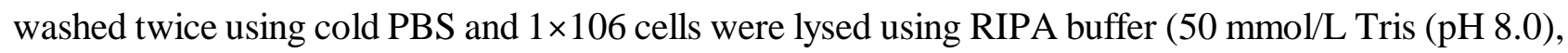
$150 \mathrm{mmol} / \mathrm{L} \mathrm{NaCl}, 0.1 \% \mathrm{SDS}, 1 \% \mathrm{NP} 40$ and $0.5 \%$ sodium deoxycholate) containing protease inhibitors (1\% cocktail and $1 \mathrm{mmol} / \mathrm{L}$ PMSF). Total proteins were separated using $15 \%$ SDS-PAGE and were transferred to a PVDF membrane. The membrane was blocked using Tris-buffered saline

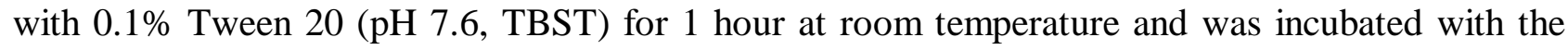
primary antibody solution $(1: 1000)$ at $4^{\circ} \mathrm{C}$ overnight. After two washes in TBST, the membrane was incubated with the HRP-labeled secondary antibody (Santa SC-2073) for 1 hour at room temperature and was washed three times with TBST. The final detection was performed using enhanced chemiluminescence (ECL) western blotting reagents (Amersham Biosciences, Piscataway, NJ) and the membrane was exposed to Lumi-Film Chemiluminescent Detection Film (Roche). Loading differences were normalized using a monoclonal B-actin antibody. The antibodies used in the study included P300 (Santa SC-585) and B-actin (Santa SC-2021)[6].

Statistics SPSS software was used for statistical analyses and the values are presented as the means +/- SD. ANOVA was used to compare the mean values. $\mathrm{P}$ values of less than 0.05 were taken to indicate statistically significant differences.

\section{Results}

Morphological changes of PC12 cells and OGD for in vitro ischemia. PC12 cells were treated with DMEM followed by the incubation for 16 hours in DMEM containing $1 \mathrm{mmol} / \mathrm{L} \mathrm{Na} \mathrm{Na}_{2} \mathrm{O}_{4}$ under hypoxic conditions $\left(37^{\circ} \mathrm{C}, 5 \% \mathrm{CO}_{2}\right.$ and $\left.95 \% \mathrm{~N}_{2}\right)$ in the absence of sugar. The cultures were photographed with a phase contrast microscope. (scale bar=20um), Values indicated are means \pm s.d. of results from four microscopic fields and asterisks indicate the statistical significance determined by unpaired t-tests $(\mathrm{P}<0.01)$.

NGF activated PC12 cells in culture. The application of the final concentration of $100 \mathrm{ng} / \mathrm{mL}$ model NGF induced PC1 2 cells $0 \mathrm{~d}, 1 \mathrm{~d}$ and $2 \mathrm{~d}, 3 \mathrm{~d}, 4 \mathrm{~d}, 5 \mathrm{~d}, 6 \mathrm{~d}$, using inverted fiber lens for morphological observation, observation results are shown in figure 2:1 d model NGF stimulation PC12 cells, can see the cells grow shorter synapse. $3 \sim 4 \mathrm{~d}$ model NGF induced PC12 cells, the length of the synaptic increase; $5 \sim 6 \mathrm{~d}$ model NGF induced PC1 2 cells, the length of the synapse can be up to $4 \sim 5$ times that of the cell body, a woven mesh, for a typical nerve cell morphology, accounts for over $90 \%$ of the total.

PC12 cells were pretreated with $\mathrm{NGF}(100 \mathrm{ng} / \mathrm{mL})$ for 1, 2, 3, 4, 5, or $6 \mathrm{~d}$. The differentiated cells were photographed under a phase contrast microscope. PC12 cells were pretreated with NGF(100 $\mathrm{ng} / \mathrm{mL})$. PC12 cells obtained from four cell depositories yielded essentially the same results. PC12 cells were exposed to the indicated concentrations of NGF for $3 \mathrm{~d}$, and the differentiated cells were counted. Significant differences were detected. PC12 cells were treated NGF $6 \mathrm{~d}$. The cultures were photographed under a phase contrast microscope (scale bars $=20 \mathrm{um}$ ), Values are means \pm s.d. of results from four microscopic fields. Asterisks indicate the statistical significance as determined by unpaired t-tests $(\mathrm{P}<0.01)$.

MAP2 immunofluorescence staining. Model NGF $(100 \mathrm{ng} / \mathrm{mL})$ induced PC12 cells into nerve cells 6d, MAP2 immunofluorescence staining results as shown in figure 2, figure 2 - A, visible under the excitation of blu-ray, star shaped scattered distribution has A long protrusions green fluorescent area, contrasts and background; The nuclei are blue fluorescent Hoechst 33258 redyeing is visible, as shown in figure 2-B; Green fluorescence image fusion after visible area for PC12 cells into nerve cells, expression of MAP2 positive control group were negative. As shown in figure 1. (A) anti-MAP2 and 
FITC-labeled IgG staining under the blue excitation. (B) nuclear counter staining of Hoechst 33258 under purple excitation. (C) merged image of the FITC and Hoechst 33258 staining.

Morphological changes captured with fluorescence microscopy following immuno-fluorescence staining ( $\times 200)$. PC12 cells were cultured with NGF for $6 \mathrm{~d}$ and were then assessed with MAP2 immunofluorescence staining.
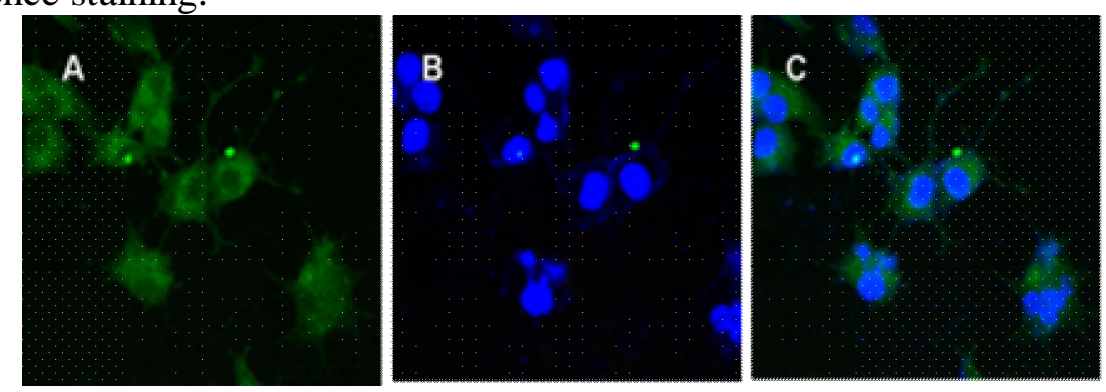

Fig.1. Immunofluorescence staining for MAP2 of NGF activated PC12 cells

Morphological changes captured with fluorescence microscopy following immuno-fluorescence staining $(\times 400)$. PC12 cells were cultured with NGF for $6 \mathrm{~d}$ and were then assessed with MAP2 immunofluorescence staining. (A) anti-NSE and FITC-labeled IgG staining under the blue excitation. (B) nuclear counter staining of Hoechst 33258 under purple excitation. (C) merged image of the FITC and Hoechst 33258 staining.

Western blotting analysis. Using Western blotting analysis model NGF induced PC12 cells into the nerve cell culture cell MAP2 protein expression changes in different time points, as shown in figure 2, model NGF induced after $3 \mathrm{~d}$, compared with control group, the expression of MAP2 protein bands thicken significantly. With the extension of model NGF stimulation time, the expression of MAP2 protein increasing trend. Is further evidence that the amount of MAP2 protein expression related to model NGF induced time, strong specificity.

PC12 cells were treated with NGF. MAP2 expression was determined by western blotting after different treatment time periods. The results shown are representative of three repeated experiments. NIH imaging indicated that the protein signal densities were increased in cells treated with NGF for 0 , 2, 4, or $6 \mathrm{~d}$ compared with control cells (no treatment). Results represent means \pm SEM for three independent observations. $\mathrm{P}<0.01$ vs. sense control (Newman-Keuls test, ANOVA)

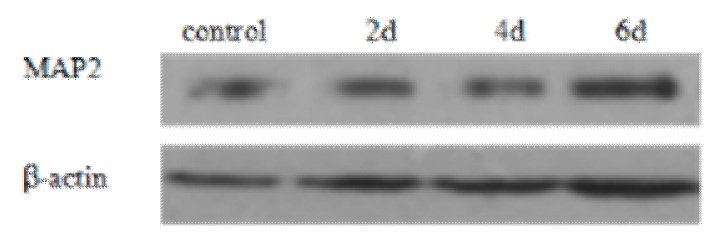

Fig. 2. Western blot analysis of MAP2

PC12 cells were treated with NGF. MAP2 expression was determined by western blotting after different treatment time periods. The results shown are representative of three repeated experiments. NIH imaging indicated that the protein signal densities were increased in cells treated with NGF for 0 , 2, 4, or $6 \mathrm{~d}$ compared with control cells (no treatment). Results represent means \pm SEM for three independent observations. $\mathrm{P}<0.01$ vs. sense control (Newman-Keuls test, ANOVA).

\section{Discussion}

Ischemic stroke is a serious harm human health disease, its mortality and morbidity is high and explore its pathogenesis and effective prevention and treatment has been the hot topics in the study of medical 
workers. In ischemic cerebral apoplexy, in order to look for can be applied in clinical nerve protection material targets and key role in signal transduction pathways, molecular level research [7].

The rat adrenal pheochromocytoma (PC12) cell line, is set up by the rat adrenal pheochromocytoma separation of cell lines, are widely used in the study of neurobiology. Learned through the literature available model NGF induced PC12 cells into nerve cells and PC12 cells can also be OGD [8], thus, can we try to combine the two, by the model NGF stimuli induced PC12 cells to differentiate into nerve cells, then to OGD of differentiated cell, to establish a repeatable nerve cells in the ischemic model. This part of the research, mainly is to create a repeatable ischemia hypoxia model of nerve cells, we use of PC12 cells, the model NGF $(100 \mathrm{ng} / \mathrm{mL})$ stimulation induced after $6 \mathrm{~d}$ to the nerve cell differentiation, differentiation of PC12 cells in the physiological and biochemical aspects has the function of the nerve cells, and induced after $6 \mathrm{~d}$ OGD, build OGD model of nerve cells in vitro.

MAP2 is one of the neuronal cytoskeleton protein [9], mainly exist in the neuronal cell bodies and dendrites, and tubulin microtubule formation, and with the other original structure such as actin, microfilament interactions constitute the cytoskeleton, participate in enation, cytoplasm protein transport, neuron model, etc. MAP2 exists in PC12 cell differentiation process, it has to do with the length of the synaptic and closely related to the formation of microtubules, MAP2 in neurons synaptic growth plays an important role in [6-9].

In the present study, we use the immune fluorescence to detect the expression of MAP2 and Western blot detection model NGF stimuli changes the expression of MAP2 in PC12 cells in different time points, the results showed that the expression of MAP2 with increasing trend with the extension of NGF plays the induction time. Description model NGF induced PC12 cells to differentiate into nerve cells, the protein expression of MAP2 in dynamic change. This provides the study of ischemic cerebral apoplexy cell basis.

\section{Acknowledgments}

The project supported by educational department and technology department of Jilin province in China. (No. 2015153, 2013ZC029, 201339033). Chunli Mei is the corresponding author.

\section{References}

[1] Kumar Mr, Bhat. Transcriptional regulation of human MAP2 gene in melanoma: role of neuronal bHLH factors and Notch1 signaling, Nucleic acids research. 34(2006) 3819 -3832.

[2] Larsenaec. Effect of D609 on phosphorlipid metabolism and cell death during OGD in PC12 cells, Neuroscience. 3 (2007) 946-961.

[3] Jae Young Lee. Neuroactive conducting scaffolds: nerve growth factor conjugation on active functionalized polypyrrole,J. R. Soc.6( 2009) 801-810.

[4] Kojike Gami. TTLL7 is a mammalian tubulin poly glutamy- lase required for growth of MAP2-positive neurites, The journal of biological chemistry.41( 2006)30707-30716.

[5] M.Katarzyna, Inhibition by an damide of 6 hydroxy-dopamine induced cell death in PC12 cells, International journal of cell biology.8( 2010)849-855.

[6] C.A.Damian,Genetos. Oxygen tension modulates neurite outgrowth in PC12 cells through a mechanism involving HIF and VEGF, J Mol Neurosci.40 (2010)360-366.

[7] Burton Tb. L. Transform growth factor induced transcription of the Alzheimer beta-amyloid precursor protein gene involves interaction between the CTCF-complex and Smads, Biochem Biophys Res Commun.3( 2002) 713-723. 
[8] A.Mirmhafieya, TGF-beta an a promising option in the treatment of multiple sclerosis, Neuropharmacology.56( 2009) 929-936.

[9] W.Sharmak, Transform growth factor- $\beta 1$ inhibits type I inositol1, 4, 5-trisphosphate receptor expression and enhances its phosphorylation in mesangial cells, J Biol Chem. 272(1997) 14617-14623. 